\title{
Estrategias para la suplementacion becerros lactantes
}

Supplementation strategies for lactating calves

Cabrera Núñez Amalia ${ }^{1 凶}$, Lammoglia Villagomez Miguel Ángel ${ }^{1}$, Iliana Del Carmen Daniel Rentería ${ }^{1}$, Díaz Inocencio Diana Laura ${ }^{1}$ y Pablo Elorza Martínez ${ }^{1}$.

${ }^{1}$ Universidad Veracruzana, Facultad de Ciencias Biológicas y Agropecuarias. Carrt. Tuxpan-Tampico Km. 7.5. Tuxpan; Veracruz; México. Tel:(01) 78383 44350. E-mail: ; malammoglia@uv.mx; idaniel@uv.mx; pelorza@uv.mx

Autor para correspondencia: amacabrera@uv.mx

Recibido: $21 / 04 / 2013$

Aceptado: 29/07/2013

\section{RESUMEN}

Con el objeto de evaluar el uso del suero lácteo en la suplementación de becerros lactantes y su efecto sobre la ganancia de peso, consumo y costo de crianza, se realizó un ensayo con diseño completamente al azar. Se utilizaron 25 becerros de la cruza Suizo/Americano - Cebú/Holstein entre 8 y 12 semanas de vida; que fueron asignados, bajo un diseño completamente aleatorio en cinco tratamientos experimentales de cinco animales cada uno; T1: 4.5 L. de suero lácteo + $1.5 \mathrm{~L}$. de sustituto/día +250 gr. de concentrado; $\mathrm{T}_{2}: 5.0 \mathrm{~L}$. de suero lácteo $+1 \mathrm{~L}$. de sustituto/día +250 gr. de concentrado; T3: 5.5 L. de suero lácteo + 0.5 L. de sustituto/día + 250 gr. de concentrado; T4: 6 L. de suero lácteo / día + 250 gr. de concentrado; T5 : 6 L. de sustituto/día. El concentrado ofrecido tuvo un $20 \%$ de proteína cruda. La duración del ensayo fue de 90 días, observándose los siguientes resultados para T1, T2, T3, T4, T5 respectivamente: Se presentaron diferencias significativas $(\mathrm{P}<0.05)$, tanto para la ganancia diaria de peso 610, 417, 436, 565 y 386 g/animal, como en la incidencia de diarreas 4\%, 12\%, 4\%, 4\%, 20\%, el análisis económico arrojó que es factible utilizar el $\mathrm{T}_{4}$ tomando en cuenta que el precio del suero es de $\$ 0.30 / \mathrm{kg}$, de esta manera el ingreso bruto se verá incrementado y por ende la relación costo/beneficio.

Palabras clave: Suplementación, Suero Lácteo, Becerros. 


\begin{abstract}
In order to evaluate the use of whey supplementation of lactating calves and its effect on weight gain, consumption and cost of breeding, a trial was conducted with a completely randomized design. 25 calves were used in the crosses Swiss / American - Cebu / Holstein between 8 and 12 weeks old, they were assigned under a completely randomized design in five experimental treatments of five animals each, T1: 4.5 L. whey + 1.5 L. substitute / day +250 gr. Concentrate, T2: 5.0 L. whey +1 L. substitute / day + 250 gr. Concentrate; T3: 5.5 L. Whey + 0.5 L. substitute / day + 250 gr. Concentrate; T4 6 L. whey / day + 250 gr. Concentrate; T5 6 L. substitute / day. Offered concentrate had a $20 \%$ crude protein. Trial duration was 90 days, observed the following results for T1, T2, T3, T4, T5 respectively: Significant differences $(\mathrm{P}<0.05$ ) for both daily weight gain 610, 417, 436, 565 and $386 \mathrm{~g} /$ animal, as in the incidence of diarrhea $4 \%, 12 \%, 4 \%, 4 \%, 20 \%$, the economic analysis showed that it is feasible to use the T4 considering that the price whey is $\$ 0.30 / \mathrm{kg}$, thus the gross income and therefore vera increased the cost / benefit.
\end{abstract}

Keywords: Supplementation, whey, Calves.

\section{INTRODUCCIÓN}

El Estado de Veracruz produce 650 millones de litros de leche al año, de los cuales aproximadamente 260 millones se utilizan en la producción de quesos. De esta industria se eliminan 235 millones de litros de suero de leche, los que son desechados a mantos acuíferos y suelo causando problemas de contaminación no cuantificados. El suero lácteo tiene un alto valor nutritivo en la alimentación de becerros por su contenido de lactosa y proteínas. Si se utilizaran los 644 mil litros de suero de leche que se producen diariamente, alcanzaría para ofrecer 4 litros diarios a 161 mil becerros de la ganadería de leche y doble propósito del estado de Veracruz. Un beneficio adicional para el ganadero es que se puede ahorrar un $25 \%$ de leche (equivale a un litro de leche diario, que en lugar de ser consumido por el becerro, se podría destinar para consumo humano) o $40 \%$ de concentrado en la crianza de becerros hasta los 90 días de edad, lo que representa $30 \mathrm{~kg}$ de concentrado, (Hernández, 2004).

En la zona donde se realizó este trabajo se observa que las explotaciones lecheras usan como sistemas de crianza de terneros el suministro de considerables cantidades de leche durante un largo período (6 a 8 meses), determinado por el sistema tradicional de ordeño "vaca-becerro". Esta alimentación es de un alto valor biológico para el becerro, pero económicamente desventajosa para el productor, ya que viene a ser competitiva con el hombre (Ugarte y Preston, 2003).

La suplementación con suero de lácteo, presenta una alternativa para mejorar los niveles nutricionales, costos de producción y el comportamiento productivo de los becerros. El suero lácteo contiene un poco más del $25 \%$ de las proteínas de la leche, cerca del $8 \%$ de la materia grasa y cerca del $95 \%$ de la lactosa, por otro lado la capacidad de amortiguamiento (la

Revista Científica Biológico Agropecuaria Tuxpan 1 (1) 
variación del PH como función de las cantidades añadidas de ácidos y bases fuertes) de un suero lácteo está determinada principalmente por las concentraciones de lactato y fosfato (Suarez et al. 1995). La búsqueda de sistemas de alimentación no convencionales ha generado en la práctica importantes resultados, las cuales reúnen y redoblan sus esfuerzos en investigación con el objeto de identificar la información básica necesaria para mejorar los diversos procesos de producción bovina típicos de nuestro medio.

El presente proyecto pretende hacer parte de estos grupos de investigación los cuales tienen como objetivo elaborar sistemas de alimentación no convencionales que posean muy buena calidad y que reduzcan los costos de producción.

\section{MATERIAL Y MÉTODOS}

El Trabajo se realizó en las instalaciones del Rancho "El Ensueño", ubicado a 98 10' 00' 'Longitud Oeste, $20^{\circ} 48^{\prime} 00^{\prime}$ 'Latitud Norte en el Municipio de Chicontepec, Verarcruz. Para observar la respuesta productiva de becerros lactantes y evaluar el costo de crianza, consumo y ganancia de peso, en un periodo de 90 días, se instaló un ensayo con 25 becerros de la cruza Suizo-Americano, Cebú -Hostein, de 8 -12 semanas de vida, los que fueron sometidos a un sistema de crianza artificial descrito en el Cuadro 1. Estos animales fueron seleccionados aleatoriamente en cinco tratamientos experimentales de cinco animales cada uno, quedando los tratamientos de la siguiente forma: $\mathrm{T}_{1}$ : $4.5 \mathrm{~L}$. de suero lácteo $+1.5 \mathrm{~L}$. de sustituto / día + 250 gr. de concentrado; $\mathrm{T}_{2}: 5.0$ L. de suero lácteo +1 L. de sustituto/día +250 gr. de concentrado; T3: 5.5 L. de suero lácteo + 0.5 L. de sustituto / día +250 gr. de concentrado; T4 : 6 L. de suero lácteo / día + 250 gr. de concentrado; T5 : 6 L. de sustituto de leche / día + 250 gr. de concentrado. Todos los becerros del ensayo, pastaron en praderas establecidas con zacate guinea (Panicum maximum) y el concentrado ofrecido presentaba el $20 \%$ de proteína cruda y 2.5 de Mcal. de energía total $/ \mathrm{kg}$.

Cuadro 1. Sistema de alimentación empleado en la suplementación de becerros.
Edad
Sistema de alimentación
Cantidad

\begin{tabular}{lcc}
\hline 1-3 difas & Calostro & Libre Acceso \\
4 días - 8 semanas & Sustituto Lácteo & 6 Litros Diarios \\
$8-12$ semanas & Suero+Sustituto Lacteo+Concentrado * & 6 Litros Diarios $+250 \mathrm{gr}$
\end{tabular}

* La cantidad de cada uno depende al tratamiento utilizado. 


\section{RESULTADOS Y DISCUSIÓN}

En el Cuadro 2 se presenta la composición química de los distintos insumos alimenticios utilizados. Los valores obtenidos del suero lácteo fueron superiores a los reportados por Raja et al. (2000) quienes reportan concentraciones de PC y NDT de 11,3 y 78,61\%, respectivamente). En cuanto a los suplementos, las mayores variaciones se observan en el nivel de PC y EE (12,23; 20,01; 2,01 y $14,34 \%$ ) para el alimento comercial y sustituto lácteo.

Cuadro 2. Composición química del suero lácteo y suplementos utilizados en la alimentación de los becerros.

\begin{tabular}{ccccccccc}
\hline Componente & MS & CEN & PC & EE & FC & ELN & NDT & HUM \\
\hline Lacto Suero & 7,06 & 5,00 & 13,93 & 22,26 & 0,15 & 62,74 & 64,62 & 92,94
\end{tabular}

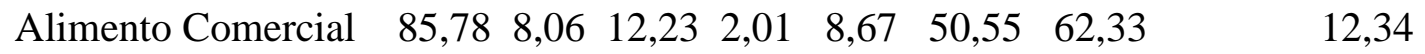

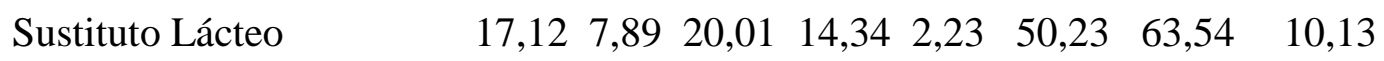

*Materia Seca (MS), Cenizas (CEN), Proteína Cruda (PC), Extracto Etéreo (EE), Fibra Cruda (FC), Extracto Libre de Nitrógeno (ELN), Total de Nutrientes Digestibles (TND), Humedad (HUM).

En el Cuadro 3 presenta los promedios de peso inicial, ganancia total y ganancia diaria de peso. Aunque la ganancia diaria de peso, no reveló diferencias entre los tratamientos $\mathrm{T}_{2}, \mathrm{~T}_{3}$, $\mathrm{T}_{4}$ y $\mathrm{T}_{5}$, los valores de este parámetro para ambos tratamientos fueron significativamente inferiores $(\mathrm{P}<0,05)$ cuando se compararon con el obtenido por los animales del $\mathrm{T}_{1}(417,436$, 565 y 386 vs 610 g/anim./día respectivamente). Estas diferencias parecen ser una consecuencia del aporte proteico, tanto del sustituto lácteo $(1,5, \quad 1,0, \quad$ vs $0.5 \quad$ lts./anim./día, para los tratamientos $\mathrm{T}_{1}, \mathrm{~T}_{2}$ y $\mathrm{T}_{3}$, respectivamente), como el aporte de 250 gr de concentrado/animal/día, para los $\mathrm{T}_{1}, \mathrm{~T}_{2}, \mathrm{~T}_{3}, \mathrm{~T}_{4} \mathrm{y}$
T5 corroborando lo obtenido por Alfani (1996). Belloso et al. (1987) y Plaza y Hernández (1994), indican que la inclusión de una pequeña cantidad de energía disponible y proteína de buena calidad a través del alimento concentrado, favorece la multiplicación y el mantenimiento de un sistema microbial ruminal más eficiente y un mejor comportamiento en su crecimiento. Iguales resultados fueron obtenidos por Labbé et al. (1985), mientras que Suárez y Benezra (1995) estudiando tres modalidades de cría de becerros doble

$\begin{array}{lllll}\text { propósito } \quad(0, & 1 & \mathrm{y} & 2\end{array}$ amamantamientos/anim./día), establecieron que el crecimiento de los becerros fue

Revista Científica Biológico Agropecuaria Tuxpan 1 (1)

ISSN: 2007-6940 
estadísticamente proporcional al número de amamantamientos.

Con respecto a la ganancia total de peso a los 90 días, los valores obtenidos fueron de 56,2; 38,$4 ; 40,2 ; 52,0$ y $35,2 \mathrm{~kg} /$ animal, para los tratamientos $\mathrm{T} 1, \quad \mathrm{~T} 2, \quad \mathrm{~T} 3, \quad \mathrm{~T} 4 \quad \mathrm{y} \quad \mathrm{T} 5$ respectivamente. La comparación de la media entre grupos, no mostró significancia estadística entre $\mathrm{T} 1$ y $\mathrm{T} 4$; siendo estas diferencias significativas $(\mathrm{P}<0,05)$. Los resultados sugieren que el reemplazo de la alimentación tradicional (sustituto de leche) por una suplementación con suero lácteo y alimento balanceado equivalente al 0,5 y $1 \%$ del peso vivo sin amamantamiento resulta en un aumento de la tasa de crecimiento, siendo esta significativamente superior $(\mathrm{P}<0,05)$ con el 1\%. Similarmente, Cazares (1979) reporta que terneros que recibieron un nivel más bajo de alimento concentrado fueron los que menos peso ganaron. Por su parte, Belloso (1986) y Alfani et al. (1996) encontraron diferencias significativas para las ganancias de peso en la medida que se incrementó el nivel de suplementación con concentrado. El efecto del sexo resultó significativo $(\mathrm{P}<0,05)$ a favor de los machos, resultados que concuerdan con los reportados por Cazares (1992) en un experimento con becerros hasta los 12 semanas de vida.

Estos resultados señalan que la alimentación a base de suero lácteo y concentrado, aportan la cantidad adecuada de proteína por consumo, reflejándose sobre la ganancia de peso vivo. Según Annison (2001), una carencia grave y contínua de proteína en animales en crecimiento, afecta la retención de nitrógeno y por lo tanto, el crecimiento animal. Por otro lado, la diferencia entre sexo debida al consumo no fue significativa, coincidiendo con Alfani et al. (1996) quienes midieron el consumo semanal de concentrado en hembras y machos, concluyendo que no hubo diferencias significativas para la variable estudiada. La suplementación con concentrado a becerros en crecimiento aumenta sus tasas de ganancia en peso. Sin embargo, forzar a estos animales a un rápido crecimiento a través de grandes cantidades de concentrado en la ración, no es con frecuencia, una práctica económica.

Cuadro 3. Comportamiento productivo de los becerros sometidos a cinco niveles de suplementación.

\begin{tabular}{|c|c|c|c|c|c|c|}
\hline Variables & & T1 & $\mathbf{T} 2$ & T3 & T4 & T5 \\
\hline $\begin{array}{l}\text { Consumo de } \\
\text { Lácteo }\end{array}$ & Suero & 2700 LTS a & 2700 LTS a & 2700 LTS a & 2700 LTS a & \\
\hline $\begin{array}{l}\text { Consumo } \\
\text { sustituto lácteo }\end{array}$ & de & $103.5 \mathrm{~kg} \mathrm{~b}$ & $69 \mathrm{~kg} \mathrm{a}$ & $34.5 \mathrm{~kg} \mathrm{a}$ & ---------- & $414 \mathrm{~kg} \mathrm{~b}$ \\
\hline $\begin{array}{l}\text { Consumo } \\
\text { concentrado }\end{array}$ & de & $115 \mathrm{~kg} \mathrm{~b}$ & $115 \mathrm{~kg} \mathrm{~b}$ & $115 \mathrm{~kg} \mathrm{~b}$ & $115 \mathrm{~kg} \mathrm{~b}$ & \\
\hline Peso inicial (kg) & & $62.2 \mathrm{a}$ & $61.8 \mathrm{a}$ & $58.6 \mathrm{~b}$ & $55.2 \mathrm{~b}$ & $61.2 \mathrm{a}$ \\
\hline Peso final (Kg) & & $119.4 \mathrm{a}$ & $100.2 \mathrm{a}$ & $98.8 \mathrm{~b}$ & $107.2 \mathrm{a}$ & $96.8 \mathrm{~b}$ \\
\hline $\begin{array}{l}\text { Ganancia de } \\
\text { acumulada }(\mathrm{Kg})\end{array}$ & peso & $56,2 \mathrm{a}$ & $38,4 \mathrm{~b}$ & $40,2 \mathrm{~b}$ & $52 \mathrm{a}$ & $35.6 \mathrm{~b}$ \\
\hline
\end{tabular}




\begin{tabular}{llllll}
\hline $\begin{array}{l}\text { Ganancia diaria de } \\
\text { peso (gr) }\end{array}$ & $610 \mathrm{a}$ & $417 \mathrm{~b}$ & $436 \mathrm{~b}$ & $565 \mathrm{a}$ & $386 \mathrm{~b}$ \\
\hline
\end{tabular}

"Valores con letras distintas en la misma fila difieren estadísticamente $(\mathrm{P}<0,05)$.

Se observó en el Cuadro 4, que el costo de alimentación fue más elevado en el T5, bajando en el $\mathrm{T}_{4}$ para aumentar ligeramente en $\operatorname{los} \mathrm{T}_{1}, \mathrm{~T}_{2}, \mathrm{~T}_{3}(5,797.00 ; 5,174.25 ;$ y $4,476.50$ Bs/anim.), indicando que el consumo de suero lácteo es más determinante $(\mathrm{P}<0,05)$ en los costos de alimentación que los niveles de suplementación, como se aprecia en el Cuadro 5 , presentando un costo unitario de $\$ 0,30$. Estos valores coinciden con los reportados por Kumar et al. (2000) quien al comparar los efectos de dos niveles de suplementación con suero lácteo (1 y 1,5 lts/anim./día) y dos edades de destete (45 y 75 días) sobre el crecimiento de becerros, obtuvo un menor costo por kilogramo de ganancia de peso para el grupo 2, el cual representó el nivel alto de suplementación. Ahuja et al. (1991), reportan que la mayor ganancia se obtuvo en los tratamientos con los niveles del $1 \% / \mathrm{kg}$ de peso, de suplementación para becerros en crecimiento.

Cuadro 4. Comparación económica de cinco niveles de suplementación en becerros.

\begin{tabular}{lccccc}
\hline \multicolumn{1}{c}{ Variables } & T1 & T2 & T3 & T4 & T5 \\
\hline Ganancia de peso total $(\mathbf{k g})$ & 56,2 & 38,4 & 40,2 & 52 & 35,6 \\
$\begin{array}{l}\text { Costo total de alimentación (Bs) } \\
\begin{array}{l}\text { Costo total para producir 1 kg de } \\
\text { carne }\end{array}\end{array}$ & $5,797.00$ & $5,174.25$ & $4,476.50$ & $3,803.75$ & $11,191.75$ \\
\hline
\end{tabular}

Cuadro 5. Costo de la suplementación empleada en los becerros de 8-12 semanas de vida.

\begin{tabular}{lccc}
\hline \multicolumn{1}{c}{ Variable } & Cantidad & Costo unitario & Costo Total \\
\hline Suero de leche (lts) & 10,800 & 0,30 & $3,240.00$ \\
Sustituto lácteo (lts) & 621 & 19,50 & $12,109.50$ \\
Concentrado comercial $(\mathrm{kg})$ & 575 & 4,25 & $2,443.75$ \\
\hline
\end{tabular}

Revista Científica Biológico Agropecuaria Tuxpan 1 (1) 


\section{CONCLUSIONES}

La suplementación con suero de lácteo mejora la ganancia de peso de los animales en la etapa de crecimiento, cuando su alimentación está basada en pastos tropicales con una disponibilidad restringida. El máximo incremento en la productividad, expresada como crecimiento, se observó cuando se compararon las ganancias en peso de animales de $\mathrm{T}_{1}$ con $\mathrm{T}_{4}$, cuyas ganancias superaron los 500 g/día. Sin embargo, el análisis económico indica que la relación Beneficio/Costo fue

significativamente superior cuando la suplementación con sustituto de leche fue de 1.5 y 6.0 lts, tomando en cuenta que el precio del suero es de $\$ 0.30 / \mathrm{kg}$, se demuestra la factibilidad de utilizar el $\mathrm{T}_{4}$ de tal manera que el ingreso bruto se vea incrementado y por ende la relación costo/beneficio.

\section{LITERATURA CITADA}

Ahuja, L. D.; M. C, Prajapati; R. D. Bhardwaj; K. K. Vyas y T. R. Lamba. 1991. Growth of Tharpakar heifers under different intensities of grazing stress on Lasiurus sindicus pasture-dryland range. Indian Journal of Animal Sciences 41(9): 806-807.

Alfani, G.; M. Ventura; D. Esparza; D. Dean y A. del Villar. 1996. Evaluación de diferentes sistemas de alimentación en becerros mestizos lecheros. Revista de la Facultad de Agronomía (LUZ) 13: 115-134.

Annison, E. F. y D. Lewis. 2001. Metabolism in the rumen. Methuen and Co., London.

Belloso, A. 1986. Efectos de cinco niveles de suplementación en el crecimiento de terneros. Tesis de Grado. Universidad Rafael Urdaneta. Maracaibo, Venezuela. $70 \mathrm{p}$.
Cazares, C. A. R. 1992. Evaluación de dos sistemas de crianza de becerros en Ganado bovino de doble propósito en el Estado de Veracruz. Tesis de Licenciatura. Facultad de Ciencias Biológicas y Agropecuarias. Tuxpan, México. p. 89-112. https://doi.org/10.5154/r.rchsh.2014.05.022

Hernández J. L. 2004. Crianza y desarrollo de becerros en el trópico. Memoria V Foro sobre Ganado Bovino en el trópico. San Jose. Costa Rica. 2001. 143-155. https://doi.org/10.21897/mvz.347

Kumar, N.; U. B. Singh y D. N. Verma. 2000. Effect of different levels of dietary protein and energy on growth de male buffalo calves. Indian Journal of Animal Sciences 51(5): 513-597.

Labbé, S.; O. Abreu; R. Rincón y R. Urdaneta. 1985. Manejo y alimentación de los animales de reemplazo en las explotaciones lecheras. Fonaiap- Divulga 2(17): 1418.

Plaza, J. y J. Hernández. 1994. Efecto del sistema de alimentación en el comportamiento de los terneros. Revista Cubana Ciencias Agrícolas 28: 175-193.

Raja, R. H.; R. Moss; R. Murray y R. Leng. 2000. Crecimiento y metabolismo de la glucosa en becerros pastoreando pastos tropicales, Efecto de la suplementación con maíz o harina de algodón. Produccción Animal Tropical. 6: 117-128. Segunda Edicion. 113-134.

Suárez, R. y M. A. Benezra. 1995. Evaluación de tres modalidades de cría de becerros doble propósito. Memorias XIV Reunión 
ALPA- $19^{\circ}$ Congreso AAPA. Argentina. pp. 419-420.

Ugarte, J. y T. R. Preston. 2003. Rearing dairy calves by restricted suckling once or twice daily on milk production and calf growth. Revista Cubana Ciencia Agricola 6: $173-182$.

Copyright (c) 2013 Amalia Cabrera Núñez, Miguel Angel Lammoglia Villagomez, Iliana Del Carmen Daniel Renteria, Diana Laura Diaz Inocencio y Pablo Elorza Martinez

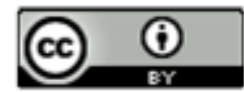

Este texto está protegido por una licencia Creative Commons 4.0.

Usted es libre para Compartir —copiar y redistribuir el material en cualquier medio of ormato- y Ad aptar el documento —remezclar, transformar y crear a partir del material- para cualquier propósito, inchso para fines comerciales, siempre que cumpla la condición de:

Atribución: Usted debe dar crédito a la obra original de manera adecuada, proporcionar un enlace a la licencia, e indicar si se han realizado cambios. Puede hacerlo en cualquier forma razonable, pero no de forma tal que sugiera que tiene el apoyo del licenciante o lo recibe por el usoque hace de la obra.

Resumendelicencia - Textocompletodelalicenria 